Publicación del Seminario "Ángel González Álvarez" de la Fundación Universitaria Española

Número monográfico sobre Mujer y cambio social Año 2021

\title{
Edith Stein (1891-1942): política y educación como herramientas de cambio social en favor de la mujer en los inicios del siglo XX
}

\author{
Edith Stein (1891-1942): politics and education as \\ tools of social change in favor of women \\ in the early 20th century
}

Milagros María MuÑOZ ARRANZ Universidad Complutense. Fac. de Educación Madrid. España

ID ORCID 0000-0001-9091-0523 milmunoz@ucm.es

Recibido: 04-08-2021 | Revisado: 20-09-2021 Aceptado: 21-09-2021 | Publicado: 30/12/2021 DOI: https://doi.org/10.51743/cpe. 228

RESUMEN: San Juan Pablo II (1999), en la Carta Apostólica en forma de Motu Proppio Spes Aedificandi para la proclamación de Edith Stein copatrona de Europa, junto con Santa Brígida de Suecia y Santa Catalina de Siena, destacó a nuestra protagonista como ejemplo de defensa de la promoción social y misión de la mujer. Se trata de una mujer polifacética que goza de un reconocido prestigio internacional dentro del ámbito filosófico y teológico. Casi un siglo después, su pensamiento no deja de sorprender y atraer. Observar las necesidades externas, aprehenderlas y procesarlas interiormente para determinar una respuesta fundamentada puede ser el resumen del 
hilo conductor que caracterizaba la actuación de Edith Stein (1891-1942). Para acercarnos a su obra, tenemos múltiples investigaciones que analizan su legado intelectual. El reto que nos planteamos en esta reflexión es desvelar su faceta más desconocida y pragmática orientada al cambio social. Para ello haremos una incursión en la actividad política y pedagógica desarrollada por nuestra protagonista en los inicios del siglo XX.

PALABRAS CLAVE: Conciencia social, educación, literatura, mujer, politización.

ABSTRACT: Saint John Paul II (1999), in the Apostolic Letter in the form of "Motu Proppio" Spes Aedificandi for the proclamation of Edith Stein co-patron of Europe, together with Saint Bridget of Sweden and Saint Catherine of Siena, highlighted our protagonist as an example of defence of both social promotion and mission of women in society. She is a multifaceted woman who has a recognized international prestige within the philosophical and theological field. Almost a century later, her intellectual thinking still surprises and attracts us. Observing external needs, apprehending them and processing them internally to decide a well-supported response can be the summary of the unifying thread that characterized Edith Stein's procedure (1891-1942). To get closer to her work, we have multiple researches about her intellectual legacy. The challenge we set ourselves in this reflection is to reveal her most unknown and pragmatic side oriented towards social change. In order to do this, we will make a foray into the political and pedagogical activity developed by our protagonist in the early twentieth century.

KEYWORDS: Women, politicization, education, social conscience, literature.

$\mathrm{E}$ 3 de agosto de 1916 se convertía Edith Stein en la primera mujer doctora en Filosofía de Alemania. Tanto el tiempo previo a este hito como los acontecimientos posteriores nos muestran a una persona decidida a hacer historia en el devenir del pueblo alemán, e incluso, en la sociedad mundial. Su biografía y su legado intelectual siguen siendo atrayentes para la sociedad del siglo XXI. La razón de tal fuerza radica en la firmeza de sus argumentos sobre la verdad de la persona, el por qué y el para qué estamos en este mundo. A sus diecinueve años se contestaba a estas preguntas: "Estamos en el mundo para servir a la humanidad, la mejor manera de hacerlo es si uno hace aquello para lo que está específicamente dotado" (Stein, 2010, p. 134). Para 
llegar a esta conclusión y poder llevarla adelante, se precisa de un equilibrado proceso de configuración de la personalidad y de una madurez espiritual que permitan la creación de una jerarquía axiológica que potencie una convivencia positiva (Muñoz Arranz, 2021).

Subrayaba que es el individuo el que tiene el deber de actuar en pro del cambio social frente al mero dejarse llevar por el vaivén de una posible sociedad moldeadora de la noción de persona para ajustarlo a los cánones del momento. En el fondo subyace la idea steiniana del respeto profundo a la dignidad humana, que implica la no valoración de la persona según criterios utilitaristas y productivos, sino apoyándose en los principios primeros que dan sentido a su existencia (Ortiz de Montellano, 2015). Con este objetivo, Edith Stein abordó la actividad política y educativa como nociones vinculadas entre sí y comprometidas con el desarrollo positivo del individuo en relación mutua con el Estado.

\section{DE LA OBSERVACIÓN DE LA REALIDAD Y LA EXPERIENCIA PERSONAL A LA APRECIACIÓN DEL CAMBIO SOCIAL COMO UNA NECESIDAD}

Edith Stein nace el 12 de octubre de 1891, en la entonces ciudad alemana de Breslau ${ }^{1}$, siendo la hermana pequeña de una extensa familia judía. El vínculo con su madre se fortaleció especialmente con la temprana y repentina muerte de su padre en 1893. La resolución materna de hacerse cargo del negocio de madera, del que eran propietarios, a pesar de la presión social y familiar, y el éxito posterior de tal emprendimiento, significaron para nuestra protagonista un ejemplo femenino de superación, decisión y valentía. Por otro lado, sus hermanos mayores ejercieron de modelos educativos sólidos que hicieron despertar en la pequeña Edith el deseo por conocer el mundo (Stein, 2010). Deseo que se materializa con la escolarización en la etapa de primaria unos meses antes de lo correspondiente por edad. La escuela y la familia se convertirían entonces en las comunidades educativas que fueron configurando una personalidad sólida

\footnotetext{
${ }^{1}$ Wroclaw en la actualidad, pertenece a Polonia tras la Segunda Guerra Mundial.
} 
y conocedora de sus intereses personales, pero al mismo tiempo sensible a las necesidades sociales (Vilanou Torrano, 2002).

Dotada de una gran vida interior, desarrollada desde los primeros años de la infancia, se cuestionó el valor de las enseñanzas adquiridas en la escuela (Stein, 2010). Su anhelo por conocer la verdad sobre la persona y el mundo, le llevaron a tomar la decisión de dejar la formación escolar tras terminar la etapa de secundaria en 1906. Los contenidos curriculares, los materiales formativos y el enfoque dado desde el sistema educativo alemán del momento decepcionaron a una joven Stein (Birkenbeil, 1990) que no buscaba un conocimiento enciclopédico heredado de la época ilustrada (Vilanou, 2002; Stein, 2015).

Entramos de esta manera en la denominada crisis de su adolescencia. Se trata de una etapa que ella misma identifica como una época de cambios profundos y fructíferos en su personalidad (Stein, 2010). Además de cuestionarse la formación recibida en la escuela, rompió con las creencias religiosas judías heredadas de la familia y asumió la razón como única fuente de conocimiento. La religión quedó relegada a un segundo plano hasta que, poco a poco, la vinculación de la persona con el fenómeno religioso iba entrando a formar parte de sus reflexiones filosóficas, pasando a ser la raíz que subyace en toda su actividad personal e intelectual a partir de su conversión al catolicismo acaecida en 1921.

Desde la Pascua de 1906 hasta marzo de 1907, su vida estuvo centrada en el cuidado de sus sobrinos y en ayudar a su hermana mayor con las tareas de la casa en la ciudad de Hamburgo (Stein, 2010). La intensa vida interior mencionada anteriormente tuvo también cabida en este período, dedicando gran parte del día a la lectura y a la reflexión personal sobre la realidad observada en aquel entorno, especialmente en lo relativo a la situación de la mujer en los inicios del siglo XX (Stein, 2010). Como vivencias que despertaron su intención de defender el valor y dignidad de la mujer, Sancho Fermín (2008) destaca:

$\mathrm{Su}$ cuñado, Max Gordon, era médico especialista en enfermedades de piel y venéreas. Hay constancia de que, en este ambiente, Edith también tomó contacto con una realidad social reflejada en algunas de las pacientes de su cuñado: mujeres contagiadas de sífilis, fruto de la doble moral de sus 
maridos. Pudo ser el detonante para el feminismo que posteriormente se radicará en Edith Stein. (p. 27).

Además de estas experiencias, empezó a ser consciente del contexto político y social presente en Alemania de los inicios del siglo XX, donde la igualdad de derechos del hombre y la mujer no eran todavía una realidad, y la educación femenina tenía carencias que solventar. Teniendo en cuenta la edad de la protagonista en estos momentos, su primera reacción surge desde un plano emocional pero sin un fundamento teórico consolidado (Muñoz Arranz, 2021). Con el estudio y la experiencia de vida fue adquiriendo una visión de la situación que ella definía como más objetiva: Como estudiante de bachillerato y joven universitaria he sido sufragista radical... Ahora busco, porque es lo que debo hacer, soluciones puramente objetivas ${ }^{2}$.

Tras un tiempo de reflexión en Hamburgo, comprendió que la mejor forma de provocar un verdadero cambio en la humanidad sería retomando los estudios: Por aquel entonces, me venía de vez en cuando el pensamiento: verdaderamente sería más sensato, que yo misma estudiase Bachillerato en lugar de colaborar ocasionalmente ${ }^{3}$.

Siguiendo este razonamiento, en septiembre de 1907, y ya de regreso en Breslau, comenzó su preparación para realizar por libre el examen de acceso a Bachillerato. Meta que alcanzó con gran éxito en abril de 1908. Fue el momento de entrar en la universidad, primero Breslau (1911-1913) y posteriormente Gotinga (1913-1915), y con ello la oportunidad de acceder a una

${ }^{2}$ Traducción propia. (Stein 2010, p. 185). «Para poder entender qué quiere decir con adoptar decisiones más objetivas, acudimos a sus conferencias sobre la mujer, donde afirmaba la necesidad de superar el entusiasmo y fogosidad de los primeros impulsos suscitados a favor de la defensa de los derechos de la mujer para retomar con calma el análisis de la situación» (Muñoz Arranz, 2021, p. 15).

3 Traducción propia. (Stein, 2010, p. 112). Con este «colaborar ocasionalmente» Edith Stein hacía referencia a la ayuda que otorgaba a su hermana Erna cuando esta cursaba su penúltimo curso escolar y ella ya no estaba matriculada en la escuela. Stein ayudó a su hermana con la elaboración de algunas de las narraciones que esta tenía que entregar como deberes de clase (Stein, 2010). Este hecho, junto con la experiencia interior respecto a la cuestión femenina expuesta anteriormente, provocó que a mediados de 1907 se despertase en nuestra protagonista un nuevo objetivo vital. 
formación acorde con sus intereses más profundos. La Psicología y la Filosofía le introdujeron en el conocimiento de la persona. Junto a ellas, destaca el estudio de la Historia y la Germanística como aquellas materias que cambiaron su forma de ver al Estado. En Edith Stein surgió un sentimiento de agradecimiento hacia aquel por haberle ofrecido la posibilidad de formarse (Stein, 2010). De esta manera, la dimensión social e individual se fue forjando como un legado recibido que debía ofrecer posteriormente siguiendo un sentimiento de responsabilidad social (Stein, 2010). Aquí se unen dos elementos principales que sitúan a Edith Stein como ciudadana activa ante el cambio social de Alemania: la importancia otorgada a una necesaria reforma de la educación en general, y de la mujer en particular; así como el fomento de la participación femenina en la vida pública.

\section{CONTRIBUCIÓN SOCIAL DE LA MUJER DESDE SU VALOR ESPECÍFICO}

Es importante tener en cuenta que, aunque la primera reacción de Edith Stein en busca de la defensa de los intereses de la mujer se haya identificado en ocasiones con una postura cercana al feminismo (Sancho Fermín, 2008), no se puede entender este concepto según la interpretación presente en la sociedad actual (García Rojo, 1998). Leyendo la introducción de su primera conferencia sobre la mujer datada el 12 de abril de 1928, El valor específico de la mujer en su significado para la vida del pueblo, podemos comprender la visión steiniana de esta noción (Stein, 2015). En esta ocasión, recordaba el eslogan presente en los inicios del movimiento feminista, emancipación, el cual se orientaba a la reivindicación de la igualdad de derechos entre hombres y mujeres, especialmente en lo relativo a la educación y el acceso a cualquier profesión (Stein, 2015).

Aunque consiguió romper moldes y doctorarse en Filosofía, Edith Stein también experimentó discriminación por el hecho de ser mujer y ser judía en sus diversos intentos por acceder a cátedra (Stein, 2002). Tras la superación del doctorado el 3 de agosto de 1916, trabajó como asistente de Husserl en la Universidad de Friburgo desde octubre de este año hasta febrero de 1918. 
Siguiendo el deseo de volcarse en la investigación filosófica, se dedicó desde entonces al trabajo privado.

El 6 de febrero 1919 sería el primero de estos intentos infructuosos de comenzar una carrera académica. Husserl, el que fuese su profesor y mentor, firmó un certificado de recomendación para la Universidad de Friburgo, aunque no llegó a presentarlo en el último momento (Stein, 2002). ¿Cuáles fueron las razones que llevaron al maestro a no presentar la carta? ¿Por ser mujer o ser judía? El que sea uno u otro motivo no dirime la existencia de prejuicios discriminatorios. El 29 de mayo de 1919, Husserl escribió a Georg Misch, profesor de Filosofía en Gotinga, presentando, esta vez sí, a Edith Stein y solicitando su habilitación en esta Universidad (Speer y Regh, 2016; Stein, 2020). En esta misma carta expresaba la razón por la que había paralizado anteriormente la recomendación para la Universidad de Friburgo:

La Señorita Dra. Stein, la cual tras su doctorado trabajó como mi asistente de cátedra durante casi dos años, desearía presentarse a usted para solicitar su recomendación respecto a la posibilidad de una habilitación en Gotinga. Permítame decirle en qué medida se trata de una valiosa personalidad que merece una atenta consideración. El que yo (no) pudiese aportar un informe de recomendación para la habilitación en Friburgo tiene, dicho en confianza, su razón, ya que en nuestra Facultad de Filosofía (que corresponde con el Departamento Filosófico-histórico de Gotinga) ya hay tres profesores de origen judío, y yo no podía esperar que fuese aprobada la habilitación de una $4^{\text {a }}$. En sí mismo, yo hubiera deseado para la asistencia de mi actividad docente una tan valiosa ayudante fenomenológica. La Señorita Stein también ha demostrado su eficacia como autora de sus propios trabajos filosóficos ${ }^{4}$.

Sin embargo, esta segunda propuesta no llegó a ser valorada oficialmente por la Universidad de Gotinga sino que fue resuelta por una precomisión formada por miembros del Departamento de Historia y Filosofía de la Facultad correspondiente (Stein, 2002). Esta vez quedaba patente que el hecho de ser mujer era el motivo de rechazo, tal y como consta en la carta enviada el 29 de octubre de 1919 a Edith Stein por el profesor Eduard Hermann: «Muy

${ }^{4}$ Traducción propia. (Speer y Regh, 2016, pp. 132-133; Stein, 2020, pp. 288-289). Texto inédito en español. 
a pesar mío, debo comunicarle que, por consejo de la comisión preparatoria, no estoy en condiciones de presentar su trabajo al encargado de dictaminar. La admisión de una dama a las oposiciones a cátedra sigue encontrando dificultades...» (Stein, 2002, p. 1418). Existen otros dos vanos intentos posteriores por acceder a la Universidad de Kiel y Hamburgo (Stein, 2002).

Los rechazos no hicieron más que consolidar su deseo de hacer patente el valor específico de la mujer como riqueza propia y para la sociedad en general (Stein, 2002). Ante la pregunta de qué entiende Edith Stein por valor específico de la mujer, García Rojo (1998) parte de la afirmación que realiza la autora en la que establece que hombre y mujer son dos subespecies diferenciadas dentro de la misma especie ser humano. Al mismo tiempo, subraya que esto no supone una gradación ni menoscabo de la dignidad de uno u otro (García Rojo, 1998).

En las conferencias y cursos impartidos desde 1928 sobre la mujer, hacía una síntesis de los rasgos definitorios de tal especificidad femenina (Stein, 2003). Como tal, destacaba la especial sensibilidad para detectar y comprender el sentir del otro, cualidad que se puede extender a la relación con la comunidad (Stein, 2003; 2015). Por otro lado añadía que, ante una misma realidad, aparece en el hombre una tendencia a posicionarse de una manera más objetiva junto con una actitud más personal por parte de la mujer (Stein, 2015). También encuentra peculiaridades a la hora de abordar el estudio o la actividad. Mientras que el hombre tiende a centrarse en la realidad que tiene delante, la mujer posee una visión más amplia (Stein, 2015). Stein (2003) resume esta diferenciación entre mujer y hombre con la expresión: «... la actitud ante lo vivo-personal frente a lo cósico...» (p. 85). Con todo ello, estas apreciaciones no suponen para Edith Stein un enjuiciamiento peyorativo, sino una percepción objetiva de la realidad (Muñoz Arranz, 2021).

Si bien es cierto que destaca estos elementos como definitorios de la naturaleza femenina y masculina, también lo es el reconocimiento de la utopía que supone pensar que el despliegue de las capacidades humanas se realizará de modo automático e igualitario en todos los individuos, recordando que existen peligros en el proceso configurativo de todo ser derivados de la convivencia de fortalezas, debilidades y carencias (Stein, 2003). De todo ello, Edith Stein argumenta la necesidad de una adecuada formación del ser 
humano en general y, teniendo en cuenta el contexto histórico en el que se pronuncia, de la mujer en particular, para contribuir eficazmente al enriquecimiento de la persona y, como consecuencia directa, obtener beneficio para toda la humanidad (Muñoz Arranz, 2021). El objetivo es alcanzar la armonía entre el yo, el tú y toda la creación.

\section{LA INTERVENCIÓN POLÍTICA DE EDITH STEIN EN LOS INICIOS DE LA REPÚBLICA DE WEIMAR (1918-1919)}

Ubicar a Edith Stein en el ámbito político nos lleva en primer lugar a una anécdota cuasiprofética que narra la protagonista en su autobiografía. Nos remontamos al año 1911 durante su último año como estudiante de Bachillerato en Breslau. Si recordamos la experiencia vivida en su crisis de la adolescencia abordada anteriormente, y por la que se sintió llamada a defender los derechos de las mujeres, queda patente en ella la presencia de una ferviente y evidente actitud luchadora. Esto explica la composición de un epigrama que se compuso para ella en la fiesta escolar de despedida de Bachillerato:

Igualdad de la mujer con el hombre

así exclama la sufragista,

con seguridad la veremos, en el Ministerio algún día

Como preludio político al paso de 1918, durante la etapa universitaria, Edith Stein formó parte de diversas asociaciones femeninas de estudiantes (Stein, 2010). Posteriormente, el 12 de noviembre de 1918, Edith Stein escribió una carta a su amigo Roman Ingarden comunicando su decisión de involucrarse activamente en la política (Stein, 2002). Para ello se alistó como miembro fundador del Deutschen Demokratischen Partei (DDP; Partido Democrático Alemán) ${ }^{6}$. Su motivación por acercarse a un partido liberal

\footnotetext{
${ }^{5}$ Traducción propia. (Stein, 2010, p. 135).

${ }^{6}$ Tal y como podemos comprobar en cuatro octavillas del mencionado partido datadas en enero de 1919 y en las que aparece el nombre de Edith Stein (Stein, 2020, pp. 280-288). El DDP es el partido antecesor del actual Frei Demokraten Partei (FDP; Partido Democrático Libre) fundado en 1948.
} 
radica en las ideas recibidas en su entorno familiar (Stein, 2010; 2020). Ella misma matiza en su autobiografía que, a raíz de estudiar Historia en la universidad, su posición frente al Estado fue evolucionando y alejándose de este liberalismo original, aunque sin llegar a los extremismos ideológicos con los que ella no estaba de acuerdo (Stein, 2010).

Dicha profundización en el conocimiento de los hechos históricos provocó en ella el crecimiento de un sentimiento de responsabilidad social al reconocer que en estos sucesos participamos todos y cada uno de los ciudadanos: «Este amor por la historia no era en mí un simple sumergirme romántico en el pasado; iba unido estrechamente a una participación apasionada en los sucesos políticos del presente, como historia que se va haciendo» (Stein, 2002, p. 302). Frecuentemente incidía en este aspecto, tal y como se puede leer en una carta enviada Ingarden 19 de febrero de 1918: «Somos nosotros quienes originamos los acontecimientos y quienes cargamos con la responsabilidad» (Stein, 2002, p. 603).

La tarea política emprendida por Stein hay que entenderla en el contexto histórico en el que tiene lugar. Con la abdicación del emperador Guillermo II, el 9 de noviembre de 1918 se puso fin al II Reich en Alemania. En ese momento comenzó a gestarse la República de Weimar, que se inauguró con las votaciones a la Asamblea Nacional el 19 de enero de 1919. Este hecho trajo como gran novedad la conquista de derechos por parte de las mujeres, entre ellos se encuentra el sufragio femenino. Ante esta situación y en virtud de su sentimiento de responsabilidad social, Edith Stein consideró que era apremiante prestar especial atención a la configuración de una dimensión social de la mujer que había estado cohibida durante muchos siglos: «Desde este sentimiento de responsabilidad me puse decididamente a favor del derecho del voto femenino» (Stein, 2002, p. 303). Objetivo que materializó cuando entró a formar parte del ya mencionado DDP (Stein, 2020). A pesar de los desengaños experimentados en este entorno ${ }^{7}$, se mantuvo fiel al compromiso adquirido hasta las elecciones de la Asamblea de la República de Weimar, previstas para enero de 1919 (Stein, 2002).

\footnotetext{
${ }^{7}$ A las pocas semanas de comunicar su incursión en política, el 27 de diciembre de 1918 volvía a escribir a Ingarden expresando su sentir: «Harta de política» (Stein, 2002, p. 669).
} 
En un primer momento, y según las octavillas del DDP de enero de 1919, su acción política, realizada a través de charlas, coloquios, entrevistas..., estaba centrada en la información y fomento de la educación de la mujer respecto a su papel activo y libre en una nueva sociedad democrática (Stein, 2020). Aunque estas intervenciones tenían como objetivo la defensa por la igualdad de derechos entre el hombre y la mujer, aprovechaba para subrayar la visión del sufragio no solo como un derecho, sino también como un deber para con la patria (Stein, 2020).

Tras la celebración de las elecciones de enero de 1919, publicó dos artículos en la revista del DDP, Der Volkstaat, en los que hacía un análisis de la actuación política de los meses precedentes (Stein, 2020). El título de ambos $\operatorname{artículos}^{8,}$ además de similares y sugerentes, permiten atisbar la orientación antropológica que sustentaba la propuesta perseguida por la autora, en la que educación y política eran dos nociones inseparables.

El primer artículo, Hacia la politización de la mujer (Zur Politisierung der Frau), fue publicado el 10 de febrero de $1919^{9}$. En él comienza reclamando la necesidad de mantener en el tiempo el cuidado del ámbito político. $\mathrm{Al}$ contrario de lo considerado por opiniones surgidas en el momento álgido de la constitución de la República, en las que se afirmaba que el asunto dejaría de tener importancia tras las elecciones, ella tenía el claro convencimiento de haberse iniciado una nueva época que no se podía obviar y escribió: «No habrá más una relación profesional, económica o cultural que no dependa de la política» (Stein, 2020, p. 11).

Denunciaba en esta publicación la peligrosa existencia de una sobreabundancia de propaganda política exaltada y mundana que había hecho daño, y que podría volver a hacerlo, tanto a los propios partidos como a la relación entre ambos y al electorado (Stein, 2020). Respecto a la misión particular de formar a las mujeres para la comprensión y ejercicio del nuevo derecho al voto, consideraba que el vertiginoso ritmo en el que había discurrido la cam-

\footnotetext{
${ }^{8}$ Los títulos y todas las citas de estos dos artículos presentes en este documento son fruto de traducción propia. Son documentos inéditos en español.

${ }^{9}$ Artículo publicado en 1919 en la revista del DDP, Der Volkstaat, n. 1. Ha sido encontrado por Carolyn Beard, en diciembre de 2018 en el Archivo de la Universidad de Breslau, Polonia (Stein, 2020).
} 
paña había impedido alcanzar los objetivos planteados: «... era casi imposible preparar al nuevo electorado político, sobre todo a las mujeres, en las inminentes tareas presentes y en la toma de posición ante los intereses profesionales y de clase que llevan al gran problema de la vida pública» (Stein, 2020, p. 11).

Respecto al papel de las mujeres en la política, destacaba en este artículo su argumento característico sobre la especificidad femenina como un valor a potenciar cuando se articula en complementariedad con el hombre: «... complementan con su idiosincrasia femenina la vida del hombre, y con él deben perseguir el fomento de la prosperidad del Estado» (Stein, 2020, p. 12). De forma particular ensalzaba la sensibilidad de la mujer, su experiencia de vida y la capacidad de reacción ante las dificultades como aptitudes sobradamente demostradas durante la época de guerras, de las que Edith Stein reconoce ser testigo, afirmando que una adecuada educación que permita el buen uso de estas disposiciones puede contribuir de forma exitosa a las metas de la democracia: «La mujer puede contribuir con éxito al verdadero objetivo democrático, es decir, lograr y consolidar la unidad del pueblo mediante el equilibrio pacífico de las relaciones de poder opuestas, solo cuando se ha formado política y socialmente» (Stein, 2020, p. 13).

Cuando habla de la necesidad de renovar la educación en su dimensión social, no está pensando solo en la mujer, sino de toda la comunidad, pues para que aquellas puedan iniciar una nueva y enriquecedora vida pública, se tornaba necesario cambiar los cánones pedagógicos seguidos hasta entonces (Stein, 2020). Se trataba de ver a las mujeres, no solo como una votante más, sino como una ciudadana en particular, como un «quien» (Stein, 2020, p. 11). Citando a Selma Lagerlö $f^{10}$, Edith Stein se cuestionaba si el Estado estaba preparado para este cambio (Stein, 2020).

Identificaba la vinculación del ámbito personal, público y profesional de cada ciudadano como la razón fundamental para comenzar a comprender la política como medio para atender, no sólo problemas del Estado, sino también asuntos de cada individuo concreto. Politizarse para adoptar una nueva

${ }^{10}$ En concreto, Edith Stein hacía referencia a Heim und Staat, conferencia impartida por Selma Lagerlöf (1858-1940), en Vortrag beim internationalen FrauenStimmrechtskongress in Stockholm, celebrado el 13 de junio de 1911(Stein, 2020). 
posición e implicación ante la realidad social (Stein, 2020, p. 11) es la expresión que usa para hablar de una nueva visión de la educación dirigida a fomentar la implicación política de cada persona, y en particular, de las mujeres. Dicho proceso formativo tendría como objetivo el desarrollo de unos valores personales que difícilmente podrían ser adquiridos por una mera regulación legislativa: «El sentimiento social, la comprensión social, el conocimiento social son los caminos que nos conducen a la verdadera democracia, a la democracia que es necesaria para la gestión tanto de la política interior como de la política exterior» (Stein, 2020, p. 21).

Como se verá más adelante, Edith Stein encontraría el camino para el desarrollo de este proceso mediante un planteamiento pedagógico fundamentado en la antropología filosófica y teológica, y apoyado por la psicología y la sociología, con el que impulsar la perfección de la persona con la ayuda de agentes externos, partiendo de la configuración interna y teniendo en cuenta las dimensiones personal, social y trascendental (Muñoz Arranz, 2021).

Poco más de un mes después del primer artículo sobre política, el 26 de marzo de 1919, volvió a publicar en la misma revista una nueva reflexión: Hacia la politización de las mujeres ${ }^{11}$ (Zur Politisierung die Frauen). Siguiendo la línea del trabajo anterior, analizaba la recién estrenada vida política de las mujeres alemanas en los primeros meses de 1919. Denunciaba, en esta ocasión, la escasa valoración que había recibido el sector político femenino debido a su poca experiencia en el ámbito (Stein, 2020). De forma elegante y elocuente respondía a aquellos que se habían acogido a tal argumentación:

... se alegó contra nosotras el hecho de nuestra falta de experiencia política. A mi no me parece que este argumento sea muestra de una gran sabiduría política, porque es sin duda una de las tareas de nuestro tiempo acuñar el tipo de mujer política, y este tipo solo puede desarrollarse en el trabajo político-práctico, en la lucha con la tarea que le ha sido encomendada. Si la concesión del derecho al voto hubiese estado condicionada a los logros políticos demostrables, todavía hoy no tendríamos un Parlamento. (Stein, 2020, p. 14)

\footnotetext{
${ }^{11}$ Artículo publicado por Edith Stein el 26 de marzo de 1919 en la revista del DDP, Der Volkstaat, n. 4. Ha sido encontrado por Waclaw Dlugoborski en 2006. (Stein, 2020, pp. 14-16).
} 
Al mismo tiempo, reconocía la premura con la que las mujeres tuvieron que enfrentarse a tareas públicas novedosas cargando con una carente formación política (Stein, 2020). Si bien esta realidad no se podía negar, y sabiendo que ante hechos similares el ser humano tiene capacidad y deber de reaccionar para solventar la situación, Edith Stein llamaba la atención para no confiarse solo en las propias destrezas naturales para atender de forma habitual los asuntos políticos (Stein, 2020). Por ello, en el artículo del 26 de marzo se dirigía a las mujeres en los siguientes términos: "Una doble tarea nos "ronda" bajo el título "Politización de las mujeres": la formación para ser ciudadana y la formación para llegar a ser líder política» (Stein, 2020, pp. 14-15). Y como líderes políticas, destacaba dos tareas: «... una era la formación de nuevas líderes; la otra se centraba en las actividades relacionadas con el Parlamento, el Gobierno y la Administración» (Stein, 2020, p. 15). Con ello concluye:

En un estado parlamentario donde la responsabilidad principal recae sobre los hombros de los representantes del pueblo, en el que el gobierno también está formado por parlamentarios, la política debe convertirse cada vez más en una profesión que no puede realizarse a tiempo parcial, sino que reclama a la persona completa y totalmente. También las mujeres que quieren dedicarse a la política... (Stein, 2020, p. 15)

Cuando aborda la formación política de la mujer, politización, incorpora nuevamente la necesidad de revisar la educación en este campo que, hasta ese momento, recibían solo los hombres:

Pero en lo que respecta a los líderes políticos, sus tareas se han incrementado tanto a raíz de la gran revolución estatal, que incluso los hombres necesitan tener una formación previa para la práctica política diferente a la que se les ofrecía hasta el día de hoy. (Stein, 2020, p. 16)

Su intervención en el mundo de la política fue breve. Desde la publicación del último artículo en marzo de 1919 no se han encontrado más datos de una actividad específica en este ámbito (Stein, 2020, pp. XLVIII-L). No obstante, resulta significativo ver que, como fruto de esta dedicación, se hace presente en sus reflexiones filosóficas la corriente pedagógica germana que considera la humanidad como un organismo vivo y a los individuos como 
miembros activos y necesarios del mismo (Muñoz Arranz, 2021). Sus trabajos posteriores, Individuo y comunidad (1919), Una investigación sobre el Estado (1920), El valor específico de la mujer en su significado para la vida del pueblo (1928), o Fundamentos teóricos de la labor social de formación (1930), son una muestra clara de la importancia otorgada a la relación individuo-sociedad como factor para la configuración de la personalidad. Consciente del peligro de caer en extremismos formativos, donde por un lado se fomenten los derechos individuales sobre los de la comunidad, o por el contrario, se relegue el valor de la persona a su capacidad de contribución efectiva al todo, se decanta por elaborar una propuesta educativa que fomente el equilibrio de intereses (Muñoz Arranz, 2021). Siguiendo estos argumentos, en noviembre de 1929, cuando estaba inmersa en el mundo pedagógico, se planteó las siguientes cuestiones relativas a la relación entre personalidad y comunidad $^{12:}$

1)¿Qué es personalidad?

2) ¿Qué es comunidad?

3) ¿Qué significado tiene la personalidad para la comunidad?

4) ¿Qué significado tiene la comunidad para la personalidad?

5) ¿Cómo deben ser configurados ambos para que se promuevan mutuamente?

Estas cuestiones, junto con la conferencia impartida el 24 de abril de 1930 Fundamentos teóricos de la labor social de formación (Die theoretische Grundlagen der sozialen Bildungsarbeit; Stein, 2003; 2004), nos ayudan a comprender la relación que establece entre la función de la escuela como educadora del individuo como tal y como miembro proactivo de la sociedad. Entonces exponía que para conseguir una sociedad viva, se tendría que configurar personalidades capaces de «vivir en comunidad y como una comunidad» (Stein, 2003, p. 131). De forma expresa, a lo largo de las intervenciones teóricas como prácticas realizadas a partir de 1923, centró

\footnotetext{
${ }^{12}$ Conferencia que no llegó a impartir. Documento hasta ahora inédito en español. Traducción propia. (Stein, 2004, pp. 169-170).
} 
la atención en la reformulación de la educación de la mujer con el objetivo de ver los resultados en la vida privada y pública.

\section{EL FOMENTO DEL CAMBIO SOCIAL DESDE LA EDUCACIÓN: EDITH STEIN COMO FORMADORA DE MAESTRAS EN ESPIRA (1923-1931)}

En abril de 1923, Edith Stein recibe una propuesta para ejercer como profesora de Literatura en el Centro de Formación de Maestras de Santa Magdalena en la ciudad alemana de Espira. Lo que en principio aparecía como una mera oportunidad de trabajo, llegó a convertirse para ella en una ocasión privilegiada desde donde investigar y llevar a la práctica sus teorías sobre la formación de la persona (Herrmann, 2012; Stein, 2020; Muñoz Arranz, 2021). Comienza así lo que se puede denominar su década pedagógica (1923-1933). Como profesora en Santa Magdalena estuvo hasta 1931, actividad que comenzó a compaginar desde 1926 con conferencias, artículos o intervenciones sobre educación en Alemania, Suiza y Austria. Finalmente, entre 1932 y 1933 trabajó como docente en el Instituto de Pedagogía Científica de Münster. Todos los trabajos realizados, tanto teóricos como prácticos, le sirvieron para proyectar la elaboración unas bases pedagógicas y, de forma particular, contribuir activamente para mejorar la educación de la mujer (Muñoz Arranz, 2021). Esta misión se vio interrumpida en abril de 1933 con la llegada del Nacionalsocialismo y la promulgación de las leyes antisemitas $^{13}$.

${ }^{13}$ La Ley para la Reconstitución del Servicio Civil Profesional (Gesetz zur Wiederherstellung des Berufsbeamtentums), promulgada el 7 de abril de 1933, determinaba que debían ser retirados de la función pública todas aquellas personas que no fuesen de ascendencia aria. El administrador del Instituto de Münster le recomendó retirarse temporalmente a la actividad privada hasta que se solucionasen los problemas políticos. Edith Stein consideró que era el momento de dejar la actividad intelectual e ingresar como carmelita en el Convento de Colonia. Desde octubre de 1933, hasta su muerte en el Campo de Concentración de Auschwitz el 9 de agosto de 1942, vivió como carmelita descalza, aunque continuó escribiendo obras de contenido especialmente teológico, algún trabajo filosófico, además de realizar traducciones y componer obras menores. No volvió a realizar ninguna contribución expresa al ámbito pedagógico, siendo la última ¿Qué es el hombre?, escrita en la primera mitad de 1933 con el objetivo de impartir un curso en el verano de ese año, algo que no llegó a suce- 
Consciente del empobrecimiento y carencias de la educación femenina en los albores del siglo XX como herencia de épocas anteriores, Edith Stein luchó por cambiar esta situación, tanto en lo que respecta a la mejora de la calidad formativa, con la recomendación de renovar los contenidos curriculares, como en la exhortación a ampliar el tiempo de escolarización de las jóvenes (Stein, 2015). En el recorrido histórico que realizó en su curso Problemas de formación de la mujer impartido en Münster en 1932, ponía en evidencia el desinterés estatal ante la formación de la mujer frente a la prestada al hombre (Stein, 2015). Unos años antes, en la conferencia de 1930 Fundamentos de formación de la mujer, denunció la falta de consistencia que habían tenido los argumentos usados en los intentos de reforma educativa en general (Stein, 2015).

La realidad histórica muestra que las primeras iniciativas en el contexto alemán, las cuales surgieron desde entornos privados, no fueron suficientes para dar respuesta a las expectativas creadas, pues los esfuerzos se centraron en la educación elemental. Frente a ello, en el citado curso pedagógico de 1932, Edith Stein se unía al clamor de un sector de la población femenina ${ }^{14,}$ que desde mediados del siglo XIX venía reclamando profesoras cualificadas para la formación de mujeres en todos los niveles y ámbitos académicos, así como para la dirección de centros de formación y la intervención en política educativa, profesiones tradicionalmente ocupadas por hombres (Stein, 2015).

Fruto de estas reivindicaciones femeninas, surgieron en Alemania los centros de formación para profesoras, entre los que se encuentran el Centro de Formación de Santa Magdalena de Espira y el Instituto de Pedagogía Científica de Münster, donde Stein ejerció como docente cualificada. A estos avances, se añaden los propuestos por el gobierno de Prusia en 1894 para el impulso de las escuelas femeninas y la reforma de 1908 que introdujo los

der por los motivos descritos anteriormente.

${ }^{14}$ En este campo, Edith Stein destaca la labor realizada por Helene Lange (1848-1930), fundadora, en 1890, de la Asociación Pública de Maestras Alemanas (Allgemeinen Deutschen Lehrerinnenverein, ADLV). Lange fue una de las primeras mujeres que advirtió que la formación estaba dirigida e impartida por hombres, y que tal hecho podría acarrear obstáculos para redefinir la educación de la población femenina. 
Liceos ${ }^{15}$ como vía de acceso a la universidad. En 1912 se promulga la Orden Ministerial para la formación de maestros y maestras que buscaba igualar los derechos en materia de educación entre hombres y mujeres.

A pesar de estos cambios, la situación de la mujer a principios del siglo XX todavía estaba sujeta a fuertes limitaciones legales. La superación de alguno de estos obstáculos, como la posibilidad de una actividad profesional reconocida y la escolarización de la mujer hasta los dieciocho años, tuvo que esperar hasta 1919 con la promulgación de la Constitución de Weimar (Stein, 2015).

Edith Stein subrayaba que el verdadero cambio requería una completa reforma del sistema educativo (Stein, 2015) que incluyese una nueva visión de la educación, valorándola como instrumento de prevención y superación de las desigualdades e injusticias sociales (Muñoz Arranz, 2021). Para ello, había que olvidarse del viejo modelo que contemplaba la educación como un proceso de trasmisión y acumulación de contenidos memorísticos por parte de un alumnado, considerado como una tabula rasa (Stein, 2015). Por el contrario, habría que potenciar el desarrollo de un espíritu crítico, con capacidad de reflexionar ante los hechos de la vida y otorgando autonomía, libertad y responsabilidad a la persona:

Las escuelas de chicas fueron creadas para transmitir ese algo indefinido que es denominado como "formación general": algo de conocimientos lingüísticos, un gran interés por la Literatura y la Historia, y cualquier otra cosa que pueda parecer apropiado para fomentar el ánimo y enardecer los

${ }^{15}$ El sistema contemplaba dos posibles vías para la formación del profesorado. La primera alternativa comenzaba con cuatro cursos de la Escuela Primara (Volksschule; de 6 a 9-10 años); posteriormente se acudía al Liceo o Instituto femenino (Lyzeum o Mädchengymnasium) durante 6 cursos más, hasta los 16 años; al terminar se podría escoger ente una Formación profesional para mujeres durante 2 años (Frauenschule), o entrar en el Liceo superior (Oberlyzeum), donde recibirían formación científica durante 3 años, más 1 seminario anual de investigación; terminando con el examen oficial de magisterio para acceder a la universidad (Lehramtsprüfung; Berg, 1991). La segunda alternativa era más práctica, más numerosa y menos elitista, que consistía en 7 cursos de Escuela Primaria (Volksschule; desde los 6 a los 13-14 años); y el acceso posterior a la Escuela de Formación o Seminario de Magisterio (Lehrerinnen-Seminar) con una duración de 6 cursos (García Garrido, 2005). Edith Stein impartió docencia en los 6 cursos de Seminario de Magisterio y en $6^{\circ}$ de Liceo (Muñoz Arranz, 2021; Stein, 2020). 
intereses; pero no hay un verdadero ámbito efectivo, ni teórico ni práctico, para desarrollar la capacidad de juicio propio y de autonomía de acción ${ }^{16}$.

En este sentido, encontró en Espira la ocasión para poner en práctica esta propuesta pedagógica (Herrmann, 2012; Muñoz Arranz, 2021), poniendo la instrucción y la enseñanza al servicio de la educación, como era su deseo (Stein, 2003). De esta intervención se desprende un qué realizó con las alumnas y un cómo intervino, es decir, qué contenidos introdujo y qué estilo docente mostró para cumplir sus objetivos.

La tradición pedagógica neohumanista, impulsada por los hermanos Humboldt en los albores del siglo XVIII con miras a la construcción de la identidad alemana a través de la cultura, acudía a los principales representantes del momento Schiller, Goethe, Lessing... (Vilanou, 2011). Los contenidos curriculares para la asignatura de Literatura determinados en la citada normativa vigente, la Orden Ministerial de 2 de agosto de 1912, seguían dicha estela impulsando el estudio de estos literatos y añadiendo, entre otros, a autores como Uhland, Geibel, Grimm, Weber, Shakespeare, Homero... Como parte de la materia se debía incluir el análisis de expresiones de uso común y refranes, así como el estudio de la historia de Alemania y, para las alumnas de último curso, la inclusión de contenidos pedagógicos (Orden Ministerial de 2 de agosto, 8 de agosto, 1912). Todo ello se completaba con la práctica de la expresión oral y la escrita, indicándose la necesidad de fomentar y respetar la exteriorización de los propios puntos de vista y valoraciones personales (Orden Ministerial de 2 de agosto, 8 de agosto, 1912). Si bien es cierto que en el debate posterior a la conferencia impartida por Edith Stein en 1930, Fundamentos de formación de la mujer, ella misma remitía a su experiencia como formadora y denunciaba que la acumulación excesiva de contenidos curriculares suponía un obstáculo para una labor verdaderamente configurativa de la persona (Stein, 2015), en la práctica docente en Espira ella supo compensar estas exigencias con su visión pedagógica, de tal forma que el uso de la Literatura como contenido curricular quedó puesto al servicio de la educación (Muñoz Arranz, 2021).

En el Archivo del Convento de Santa Magdalena de Espira, se conserva

\footnotetext{
${ }^{16}$ Traducción propia. (Stein, 2015, p. 144).
} 
un cuaderno de Edith Stein realizado durante los años en los que fue docente en este centro. En él aparecen clasificados por curso y nivel educativo los diferentes enunciados de ensayo propuestos a sus alumnas en sus clases. Junto a este documento, existen 59 hojas sueltas que contienen diferentes esquemas cuyo contenido se puede relacionar con los epígrafes recogidos en el cuaderno ${ }^{17}$ La organización y análisis de este legado nos permite comprobar la habilidad mostrada por Stein para ajustarse a lo prescrito legalmente al mismo tiempo que, aprovechando el margen de acción permitido por la normativa, introduce contenidos antropológico-filosóficos presentes en su propuesta pedagógica: la persona con sus limitaciones naturales, con capacidad y necesidad de educación; la elección consciente o inconsciente de modelos de vida hacia los que la persona dirige la acción en busca de su posible perfección; el valor de las experiencias vitales como herramienta educativa; la determinación de objetivos para el impulso de la motivación; la educación de la vida interior; la formación de la juventud para salvaguardar el futuro de la comunidad; la familia y escuela como agentes educadores; la individualidad del ser humano como riqueza a compartir con la humanidad... (Muñoz Arranz, 2021).

Stein tiene un estilo educativo propio. Como profesora de Literatura tenía que realizar una cantidad de ensayos anuales en relación con las obras leídas en clase. Con esta indicación, utilizó las obras de Goethe, Schiller, Lessing..., y todos los demás contenidos literarios, como centros de interés desde los que rescatar de ellos una cita o un aspecto determinado y, desde ahí guiar la reflexión de sus alumnas (Muñoz Arranz, 2021). Esta es la excelencia pedagógica mostrada en Edith Stein, pues fue capaz de dar un sentido más profundo a los contenidos curriculares, superando así el mero aprendizaje memorístico (Muñoz Arranz, 2021).

Dentro del amplio elenco de temas tratados por Stein, la cuestión de la mujer destaca por ser el más abordado, así como por la sutileza de su enfoque. En las propuestas de ensayo subsiste el anhelo steiniano por trasmitir a las alumnas el valor positivo de la especificidad femenina, y el fomento de

\footnotetext{
${ }^{17}$ El contenido organizado de este cuaderno y las hojas explicativas sueltas está publicado en el volumen 28 de las Obras Completas de Edith Stein en alemán (Edith Stein Gesamtausgabe; Stein, 2020).
} 
actitudes críticas y valientes para intervenir tanto en el ámbito privado como público (Herrmann, s.f.). Es, además, un ejemplo claro del modelo docente steiniano, ya que, aunque la mujer no era un contenido incluido (tampoco excluido) en la Orden Ministerial del 2 de agosto de 1912, ella lo rescató conscientemente y lo puso en relación con las obras literarias que se habían de tratar en clase.

Enfocó la atención en el análisis de los personajes femeninos y en las características de su personalidad mostradas en las circunstancias vitales que rodean a cada una de ellas, sirviéndose de obras como Guillermo Tell de Schiller y Minna von Barnhelm de Lessing (Muñoz Arranz, 2021). Para ello planteó los siguientes temas de ensayo:

- «Los personajes femeninos en la obra Guillermo Tell de Schiller» ${ }^{18}$

- «Gertrud y Werner Stauffacher» ${ }^{19}$

- «Gertrud Stauffacher. La imagen de un temperamento» ${ }^{20}$

- «El enfrentamiento de ambas reinas en la obra María Estuardo de Schiller» ${ }^{21}$

- «María Estuardo. La imagen de un temperamento» ${ }^{22}$

- «Características de una de las protagonistas de la obra de Lessing Minna von Barnhelm» ${ }^{23}$

18 Traducción propia. Enunciado propuesto a las alumnas de cuarto en el año escolar 19231924: «4. Die Frauengestallten in Schillers $<$ Wilhelm $>$ Tell.» (Stein, 2020, p. 92). Enunciado propuesto como deberes escolares a las alumnas de sexto de Liceo en el año escolar 1927-1928: «1.) a. Frauencharaktere in Schillers Willh.<elm>Tell» (Stein, 2020, p. 44).

${ }^{19}$ Traducción propia. «2.) Gertrud und Werner Stauffacher.» (Stein, 2020, p. 61). Enunciado propuesto a las alumnas de sexto de Liceo en el año escolar 1929-1930.

${ }^{20}$ Traducción propia. «2.) b. Gertrud y Werner Stauffacher. Ein Charakterbild.» (Stein, 2020, p. 68). Enunciado propuesto a las alumnas de sexto de Liceo en el año escolar 1930-1931.

${ }^{21}$ Traducción propia. Enunciado propuesto a las alumnas de tercero en el año 1923-1924: «11. Die Begegnung der beiden Königinnen in Schillers Maria Stuart.» (Stein, 2020, p. 91). Enunciado propuesto a las alumnas de cuarto como deberes escolares en el año 19301931: «I.) b. Die Begegnung der beiden Königinnen in Schillers „,Maria Stuart“.» (Stein, 2020, p. 71).

${ }^{22}$ Traducción propia: «<1.)> b. Maria Stuart. Ein Charakterbild.» (Stein, 2020, p. 51). Enunciado propuesto a las alumnas de cuarto en el año escolar 1928-1929. 
- «Kriemhild. La vida de una mujer» ${ }^{24}$

La capacidad de empatía, las cualidades intelectuales y la perseverancia ante los momentos de adversidad fueron aspectos de la personalidad de María Estuardo ensalzados por Edith Stein para fomentar la emulación entre sus alumnas (Muñoz Arranz, 2021):

El personaje de María Estuardo en el primer acto de la obra Schiller

A. El primer acto nos da a conocer la personalidad y la situación de María Estuardo

B. Nosotros la conocemos:

I. como una mujer con grandes dones intelectuales
a. erudita
b. sagaz
c. elocuente
d. sensata

II. como mujer con un corazón tremendamente vivaz y sensible;

a. con facilidad para inflamar la pasión,

b. de fiel predilección y cuidado de los suyos;

c. hija fiel de su Iglesia;

d. también amable con los extraños;

III. como mujer de fuerte y decidida voluntad:

a. en la penitencia y la lucha contra sus errores;

b. en su lucha por la liberación.

C. La descripción del primer acto suscita un vivo interés por la protagonista.

(Stein, 2020, pp. 51-52)

Consideró importante comparar la imagen de mujer en diferentes momentos históricos, e incluso en culturas diversas, para subrayar la relación

${ }^{23}$ Traducción propia. «5.) Charakteristik einer der Hauptgestalten aus Lessings ,, Minna von Barnhelm “.» (Stein, 2020, p. 68). Enunciado propuesto a las alumnas de sexto de Liceo en el año escolar 1930-1931.

${ }^{24}$ Traducción propia. «7.) Kriemhild. Ein Frauenleben.» (Stein, 2020, p. 26). Kriemhild es la protagonista femenina de El cantar de los Nibelungos. Enunciado propuesto a las alumnas de cuarto en el año escolar 1925-1926. 
existente entre ideales de un pueblo y la consideración de la población femenina. El canto de Waltarius de Ekkerhard, Ifigenia de Schiller, la obra anónima El cantar de los Nibelungos, e incluso la lectura de las mitología griega fueron las fuentes presentadas por Stein para la reflexión en clase (Muñoz Arranz, 2021):

- La vida de las mujeres en el Edad Media (según El cantar de los Nibelungos) $)^{25}$

- El ideal germánico de mujer (según El canto de Waltharius) ${ }^{26}$
A. La poesía de Ekkerhard.
B. I. Entrega humilde y fiel.
II. Coraje.
III. Misericordia.
IV. Inteligencia.
C. Aplicación o: el ideal heroico.

(Stein, 1923-1931, p. 33)

El ideal de mujer en la antigua Grecia y en la antigua Alemania ${ }^{27}$.
A. La disparidad de los pueblos se refleja en sus ideales.
B. I. Ambos valoran
- belleza
- pureza
- lealtad
II. Hay que añadir las diferencias
a. con los griegos
1. inteligencia y habilidad
2. escasa subordinación
b. con los germanos

${ }^{25}$ Traducción propia. «4.) Frauenleben im Mittelalter. (Nach dem Nibelungenlied)» (Stein, 2020, p. 19). Enunciado propuesto a las alumnas de cuarto en el año escolar 1924-1925.

26 Traducción propia. «5.) Das germanische Frauenideal (Nach dem Waltharilied).» (Stein, 2020, p. 32). Enunciado propuesto a las alumnas de cuarto en el año escolar 1927-1928.

27 Traducción propia. «<3.) > b. Altgriechisches und altgermanisches Frauenideal.» (Stein, 2020, p. 53). Enunciado propuesto a las alumnas de cuarto en el año escolar 1928-1929. 


\section{1. fortaleza corporal}

2. fuerza de la pasión

3. orgullo y voluntad de poder

C. La transformación de los ideales germánicos por la cristiandad.

1. El ideal de mujer no está concreto

2. Por los griegos ninguna consideración a la fidelidad

3. Por los germanos principalmente la valoración de la humildad y la introversión.

4. Ifigenia de Schiller

5. El peinado griego

6. Amor madre - hijo

(Stein, 2020, pp. 53-54)

Fiel a las indicaciones normativas en consonancia con las tendencias pedagógicas alemanas de inicios del siglo XX, Goethe aparece también como centro desde el que tratar estas cuestiones (Muñoz Arranz, 2021):

De la Antigüedad y lo Cristiano en la Ifigenia de Goethe

A. Ifigenia, modelo del estilo clásico.

B. I. Elementos de la Antigüedad

a. el material

b. la forma rigorista

c. la creencia en el destino en El canto de las Parcas $^{28}$

d. el carácter de Pílades ${ }^{29}$

II. Elementos cristianos

a. la relación entre los griegos y los bárbaros.

b. la fe de Ifigenia.

c. su misión redentora

${ }^{28}$ (Lied der Parzen). Las Parcas son las diosas de la mitología romana que disponían de la vida del hombre. En este punto Edith Stein está haciendo referencia al monólogo de Ifigenia en Táuride, donde aborda la relación entre los dioses y los humanos, y el fatal destino de la humanidad (Stein, 2020, p. 83).

29 (Pylades). Amigo del hermano de Ifigenia, Orestes. Pílades acompaña a Orestes hasta Táuride para rescatar a Ifigenia. 
C. Opinión propia hacia la poesía.

(Stein, 2020, p. 83)

Junto a este esquema, Edith Stein recopila en su cuaderno los siguientes ensayos propuestos a sus alumnas. En ellos aparece nuevamente el análisis de Ifigenia como ejemplo de mujer con un papel decisivo en el devenir de la historia narrada:

- «¿Cómo cumple Ifigenia con su misión?» ${ }^{30}$

- «¿Qué motivos influyen en Ifigenia para tomar su determinante decisión?» ${ }^{31}$

No faltó la ocasión abordar con sus alumnas el rol de la mujer en la comunidad y fomentar la reflexión de su misión como parte activa, con sus derechos pero también con sus deberes sociales (Muñoz Arranz, 2021):

- «Opinión de Goethe respecto a la profesión femenina y destino y vida de las mujeres en nuestro tiempo» ${ }^{32}$

- «¿Qué tareas son asignadas a la mujer de nuestro tiempo?» ${ }^{33}$

- «La misión más importante de la mujer en nuestro tiempo» ${ }^{34}$

- «Nosotras jóvenes y nuestro tiempo. ¿Qué nos pide y qué nos aporta y que le debo yo? (3 argumentaciones) $\rangle^{35}$

${ }^{30}$ Traducción propia. «7.) Wie erfüllt Iphigenie ihre Mission?» (Stein, 2020, p. 21). Enunciado propuesto a las alumnas de quinto en el año escolar 1924-1925.

31 Traducción propia. «5.) Welche Motive bestimmen Iphigenie zu ihrem entscheidenden Entschluß?» (Stein, 2020, p. 44). Enunciado propuesto a las alumnas de sexto de Liceo en el año escolar 1927-1928.

${ }^{32}$ Traducción propia: «3.) c. Goethes Auffassung von Frauenberuf und -Schicksal u. $<$ nd $>$ das Frauenleben unserer Zeit» (Stein, 2020, p. 31). Enunciado propuesto a las alumnas de quinto en el año escolar 1926-1927.

${ }^{33}$ Traducción propia: «2.) c. Welche Aufgaben sind der Frau in unserer Zeit gestellt?» (Stein, 2020 , p.44). Enunciado propuesto como deberes escolares a las alumnas de sexto de Liceo de 1927-1928.

${ }^{34}$ Traducción propia. «<3.) > b. Die wichtigsten Aufgaben der Frau in unserer Zeit.» (Stein, 2020, p. 68). Enunciado propuesto a las alumnas de sexto de Liceo en el año escolar 19301931. 

A. Cambios en la vida de la mujer
B. Las tareas de la mujer
1. En la vida doméstica
2. En la vida profesional
3. En la vida pública
a. En la vida política
b. En la vida social
C. ¿Cuál debe ser el objetivo que esté por encima de todos?

(Stein, 2020, p. 45)

\section{CONCLUSIONES}

Los argumentos políticos y pedagógicos defendidos por la autora en una época de cambios en la Alemania de los inicios del siglo XX pueden hacernos reflexionar ante el devenir de la sociedad actual. Edith Stein no contempla hablar de un verdadero cambio social si no se tiene en consideración al mismo tiempo el desarrollo de las fuerzas y disposiciones de cada persona como ser singular y en calidad de miembro de un pueblo. Puntualizaba, por lo tanto, que la sociedad es necesaria para el individuo tanto como éste para el todo. Si estos puntos de vista están enfrentados, se coartan las fortalezas individuales y se rompe la necesaria unidad de la comunidad, sin la cual no puede haber avance.

Valorando a los dos artículos expuestos, La politización de la mujer (Zur Politisierung der Frau) y La politización de las mujeres (Zur Politisierung der Frauen), queda patente cómo el principal interés de Stein era despertar el valor del nuevo derecho al voto adquirido en Alemania en 1919. Para ello establecía una relación intrínseca entre política y formación, de tal modo que la mujer pudiera insertarse proactivamente en la vida pública, entendiendo por tal, no solo una acción externa, sino buscando un movimiento interno orientado al desarrollo de una conciencia de responsabilidad social. Alerta, además, del peligro real que

\footnotetext{
${ }^{35}$ Traducción propia. «Wir Mädchen und unsere Zeit. + Was nimmt und gibt sie uns und was bin ich ihr schuldig? (3 Bearbeitungen)» (Stein, 2020, p. 86). No hay constancia de la fecha de la propuesta ni del grupo al que fue dirigida.
} 
supone caer en un comportamiento externo políticamente correcto que no contribuye a la perfección del ser ni al de la humanidad en general.

Por otro lado, su discurso para la defensa de la cuestión educativa femenina se centraba en lo que consideraba la necesidad imperante del momento: la revisión de las oportunidades educativas y profesionales de la mujer (Stein, 2015). Esta proposición podría haberse quedado en una utopía del momento si no fuera por la demostración práctica realizada por la propia Edith Stein en su intervención como docente en Espira. En este sentido, la educación fundamentada en la antropología e identificada como herramienta de prevención y promoción social por encima de la mera enseñanza, queda como un reto imperecedero que tiene que asumir la sociedad del siglo XXI: «... por eso es correcto contemplar hoy de nuevo a la educación como su tarea primordial, y a la instrucción como medio al respecto» (Stein, 2003, p. 337).

\section{REFERENCIAS BIBLIOGRÁFICAS}

Berg, C. (Ed.). (1991). Handbuch der deutschen Bildungsgeschichte. Band IV. 18701918. Von der Reichsgründung bis zum Ende des Ersten Weltkriegs. Verlag C. H. Beck.

Birkenbeil, E. J. (1990). Dr. Edith Stein: Assistentin, Lehrerin, Dozentin. En L. J. Elders SVD (Ed.), Edith Stein. Leben, Philosophie y Vollendung. Abhandlungen des internationalen Edith-Stein-Symposiums Rolduc (pp. 95109). Johan Wilheim Naumann.

García Garrido, J. L. (2005). Sistemas educativos de hoy (4.aed.). Ediciones académicas.

García Rojo, E. (1998). Edith Stein. Existencia y pensamiento. Editorial de Espiritualidad.

Herrmann OP, M. A. (2012). Edith Stein. Ihre Jahre in Speyer. Media Maria Verlag.

Herrmann OP, M. A. (s.f.). Aufsatzstunde-Lebenskunde. Gedanken zu Aufsatzthemen von Edith Stein (manuscrito sin publicar). Archivo del Convento de Santa Magdalena de Espira, Alemania.

Juan Pablo II. (1999). Carta Apostólica en forma de «Motu Proppio» Spes Aedificandi para la proclamación de Santa Brígida de Suecia, Santa Catalina de Siena y Santa Teresa Benedicta de la Cruz copatronas de Europa. http://w2.vatican.va/content/john-paul-ii/es/motu_proprio/documents/hf_jpii_motu-proprio_01101999_co-patronesses-europe.pdf 
Muñoz Arranz, M. M. (2021). La pedagogía con sentido en el pensamiento de Edith Stein: sistematización de su legado pedagógico (1926-1933) y su relación con la práctica docente como formadora de maestras en Espira (1923-1931) (Tesis doctoral). Universidad Complutense de Madrid.

Orden Ministerial de 2 de agosto de 1912. (8 de agosto de 1912). Die Bildung der Lehrer und Lehrerinnen (La formación de maestros y maestras). Ministerialblatt für Kirchen und Schulgelegenheiten im Königreich Bayern, 25, 397-466.

Ortiz de Montellano L.C., O. (2015). Edith Stein, el ethos femenino y el papel de la mujer en la enseñanza. Ecclesia. Revista de cultura católica, XXIX(3-4), 229257.

Sancho Fermín, F. J. (2008). El ambiente espiritual y humano de Edith Stein. En U. Ferrer (Ed.), Para comprender a Edith Stein (pp. 15-94). Ediciones Palabra.

Speer, A. y Regh, S. (Ed.). (2016). "Alles Wesentliche lässt sich nicht schreiben». Leben und Denken Edith Steins im Spiegel ihres Gesamtwerkes. Verlag Herder $\mathrm{GmbH}$.

Stein, E. (2002). Obras completas I. Escritos autobiográficos y cartas. Editorial Monte Carmelo.

Stein, E. (2003). Obras completas IV. Escritos antropológicos y pedagógicos. Editorial Monte Carmelo.

Stein, E. (2004). Bildung und Entfaltung der Individualität (2. ${ }^{a}$ ed., vol. 16). Verlag Herder.

Stein, E. (2010). Aus dem Leben einer jüdischen Familie und weitere autobiographische Beiträge (3. ${ }^{\mathrm{a} e d}$., vol. 1). Verlag Herder GmbH.

Stein, E. (2015). Die Frau. Fragestellungen und Reflexionen (5.aed., vol. 13). Verlag Herder GmbH.

Stein, E. (2020). Neu aufgefundene Texte und Übersetzungen VII. Texte zu Philosophie, Politik, Pädagogik; Übersetzung: Bonaventura, KarmelGeschichte, „Judenfrage“. Neu aufgefundene Briefe und Dokumente (vol. 28). Verlag Herder GmbH.

Vilanou Torrano, C. (2002). La pedagogía teológica en Edith Stein (1891-1942). Revista española de pedagogía, 60(223), 481-500. https://www.jstor.org/stable/23764653

Vilanou Torrano, C. (2011). La Pedagogía alemana y su recepción en España: la idea de formación y las ciencias del espíritu. En J. M. Hernández Díaz (Coord.), La pedagogía alemana en España e Iberoamérica (1810-2010) (pp. 135-192). Castilla Ediciones. 